\title{
Trauma nacional y cultura indígena. Los sucesos de 1932 en El Salvador
}

\author{
David Hernández \\ Universidad de Hannover, Alemania \\ hernandezdavid500@gmail.com
}

\begin{abstract}
Resumen
El presente trabajo parte del trauma nacional generado por la masacre de más de quince mil indígenas en 1932, de parte del gobierno del general Maximiliano Hernández Martínez, lo cual generó la virtual desaparición de los últimos restos de cultura indígena náhuat-pipil, principalmente su idioma, vestimentas, costumbres y tradiciones. Analiza las consecuencias de dicha masacre en la historia del país, contextualizada en las coyunturas político-económicas de la época.
\end{abstract}

Palabras clave: cultura indígena, masacre, historia político-económica.

\begin{abstract}
This work of the national trauma generated by the slaughter of more than fifteen thounsand Indians in 1932 by the government of General Maximiliano Hernandez Martínez, wich led to the virtual disappearance of the las remnants of Nahuatl-Pipil indigenous culture, especially its language, clothing, custums and traditions. Analyzes the consequences of such slaughter in the country's history, contextualized in the political and economic circumstances of the time.
\end{abstract}

Keywords: indígenous culture - slaughter - polítical and economic history

\section{Estructura y métodos}

El presente estudio se divide en tres partes principales subdivididas a su vez en capítulos. La primera parte se ocupa de los acontecimientos de 1932 como forma traumática de ingreso de El Salvador a la modernidad y hace la genealogía de los discursos de la identidad salvadoreña, a partir de esa fecha. En la segunda se analizan las búsquedas, a través de diferentes caminos, de los posibles discursos 
sobre los procesos de mestizaje, la identidad cultural y la heterogenidad hasta los años 50, como también las carencias de dichos trabajos y sus causas. En este contexto es válido situar, como un punto de especial referencia, los trabajos investigativos de Leonhard Schultze Jena, así como el hecho de que no se les haya prestado la debida atención investigativa hasta el momento. En la tercera se sitúan las reinvenciones de los elementos culturales indígenas en la literatura entre 1959 y 1979 y se reconstruye lo ocurrido con las herencias indígenas nahua y pipil y la redefinición de la identidad cultural durante la guerra civil y la actual postguerra.[1]

Estos planteamientos hacen una lectura de las huellas, así como del papel que juega la suplantación y/o represión de los hechos del 32 y su trauma histórico en todo el inconsciente colectivo de la nación.

La primera parte se abre proponiendo un examen de las cuatro interpretaciones principales de los acontecimientos del año 1932. En el occidente del país, en la llamada Región de los izalcos, una insurrección indígena enfrentó amplias capas de la sociedad salvadoreña, el recién fundado Partido Comunista, los sindicatos y parte del Ejército, al grueso del Ejército como fuerza represora del Estado oligárquico-liberal. La represión que siguió trás la derrota de la insurrección costó la vida a decenas de miles de indígenas en dicha región. Entre las principales causas que originaron la insurrección están las siguientes:

a) la injusta distribución de la tierra en El Salvador, producida por la Reforma Liberal de 1881-1882 que promovió el presidente Rafael Zaldívar, y que arrebató las tierras comunales y los ejidos a las comunidades indígenas para entregarlas a la oligarquía terrateniente que había puesto en marcha el monocultivo del café. Las comunidades indígenas fueron despojadas de sus tierras, convirtiéndose los indios en jornaleros agrícolas asalariados durante los seis meses que duraba la recolección del café (de octubre a mayo). El Salvador pasó a ser en menos de dos décadas (de 1880 a 1900) un país de monocultivo de café, cuyo principal rubro económico dependía de los precios internacionales del "grano de oro";

b) el "crac" de la bolsa de Nueva York de 1929, detonador de la primera crisis mundial capitalista. A raíz de esta, los precios del café se vinieron abajo, generando una crisis económica en El Salvador. Hambre y desempleo empujaron a los indios izalcos a su desesperado levantamiento.

Dadas esas causas y el transcurso de los acontecimientos del 32, han cristalizado históricamente, en la lucha por su interpretación, cuatro versiones que pueden condensarse en la forma que sigue:

a) la autointerpretación que proponen los protagonistas vencedores, es decir, la oligarquía y su dictadura militar. Esta interpretación esgrime el fantasma de la amenaza "comunista", asociando a las masas insurrectas indígenas a la barbarie. El estereotipo así acuñado es el de la amenaza de la "barbarie roja". En este punto es representativo el análisis y comentario del libro 
Revolución comunista. ¿Guatemala en peligro? [2] escrito por el periodista guatemalteco Jorge Schlesinger por encargo de la tiranía de Maximiliano Hernández Martínez;

b) las interpretaciones que da la historia oficial desde los años 50, incluidas en los libros de texto de la historia nacional;

c) la gran contra-interpretación que trató de proponer Roque Dalton con un texto de interpretación político-sociológica puramente narrativo: el testimonio Miguel Mármol. Esta contra-interpretación sacó a la palestra el importantísimo aspecto que juega la rebelión del 32 en la valoración que le dan las vanguardias revolucionarias armadas que surgen a partir de los años setenta en El Salvador y que conforman el grueso del FMLN que libra la guerra de liberación nacional del año 1980 hasta la firma de los acuerdos de paz en 1992;

d) La interpretación de los sucesos de 1932 como una insurrección propiamente indígena propuesta por el historiador centroamericano Héctor Pérez Brignoli en su trabajo Indios, comunistas y campesinos. La rebelión de 1932 en El Salvador. A la luz de esa interpretación se confirma una hipótesis de largo alcance: la represión de la rebelión indígena de 1932 anuló los restos de cultura indígena y acabó de unificar culturalmente (idioma español y religión católica) al campesinado salvadoreño.

Después de la "matazón del 32" las culturas indígenas salvadoreñas, en especial la de los pipiles de Izalco, pasaron a existir sólo como culturas sonámbulas, clandestinas, subversivas y prohibidas. A partir de 1932, sobre todo en el Occidente del país, hablar en nahuat, portar los trajes típicos indígenas, y en general, practicar la danza o los cantos pipiles, era una forma de exponerse a ser asesinado o cuando menos arrestado por la Guardia Nacional, la Policía de Hacienda o por las "Guardias cívicas" formadas por paramilitares, que patrullaban la región. La cultura pipil fue condenada al ostracismo, y en menos de una generación, prácticamente perdió toda su vitalidad debido a que se experimentó un proceso de "ladinización" forzada en el territorio Occidental del país. Los nombres indígenas de las familias fueron españolizados, los indios sobrevivientes de la masacre tuvieron que emigrar al Oriente del país y a Honduras huyendo de la represión, hablar en pipil era causa suficiente para ser considerado un "rojo". La oligarquía salvadoreña aprovechó el fantasma de la "barbarie comunista india" para hacer un "borrón y cuenta nueva" con las aldeas, pueblos y comunidades pipiles.

Quizás esta sea una de las causas para que al libro de Schultze Jena se le prestara poca atención: sus informantes habrían sido "los pipiles comunistas de Izalco"; Indiana Pipil recoge la herencia cultural de la "barbarie india".

No puede pasar desapercibido en este contexto sociopolítico la aparición de Cuentos de barro, en 1933, de Salarrué. Esta obra, que recoge el lenguaje coloquial español del campesino salvadoreño, presenta también en sus 
narraciones numerosos étimos pipiles y da una visión de la vida en la campiña salvadoreña a través de un español cargado de arcaísmos de la época colonial. Este libro ha devenido en un paradigma de la literatura nacional; y de ahí su importancia, ya que fue traducido al ruso, promovido por los intelectuales de izquierda, y en 1965 fue publicado en Cuba a instancias del poeta Roque Dalton. Cuentos de barro es una obra que rescata parte de "las memorias de larga duración" en el campesinado salvadoreño e incluye elementos étnicos propios de "los procesos de resistencia" salvadoreños. Decisivo es, en todo caso, uno de los reproches que se le hacen, sin embargo, a Salarrué: no mencionar en absoluto en su libro la masacre de 1932. Respecto a este reproche, hemos querido profundizar la discusión en este trabajo; y por ello nos referimos a él haciendo un balance crítico.

Por otro lado, si se piensa en el destino de las culturas étnicas en El Salvador hasta 1956, es necesario tomar en cuenta los movimientos populares que hay en Centro américa en la década de los cincuenta, y que tienen sus puntos álgidos en tres hechos fundamentales de la historia reciente:

a) el derrocamiento en El Salvador de la dictadura de Maximiliano Hernández Martínez (1931-1944) por la huelga general de brazos caídos de abril y mayo de 1944, luego del cual se abre un breve período democrático que es acallado a sangre y fuego por el golpe de Estado del coronel Osmín Aguirre y Salinas (exdirector de la Policía Nacional en 1932) en octubre de 1944. A partir del 14 de diciembre de 1948, sin embargo, con el golpe de Estado que inicia la "revolución de los mayores", surge en El Salvador un movimiento reformista encabezado por el coronel Óscar Osorio, que es favorecido por la bonanza de los precios internacionales del café, lo cual permite a los gobiernos militares salvadoreños realizar una serie de reformas sociales de carácter cosmético en los renglones de salud, vivienda y seguridad social, así como la construcción de diferentes obras de infraestructura, una de las cuales, la Presa Hidroeléctrica del Río Lempa, va a convertirse en símbolo de esta década de empuje económico y nacimiento de una industria regional;

b) el golpe de Estado contrarrevolucionario y la intervención directa de los arquitectos de "la guerra fría", los hermanos Foster Dulles, a través de la CIA y la compañía bananera United Fruit Company contra el gobierno democrático de Jacobo Arbenz en 1954 en Guatemala;

c) los procesos de descomposición de las tiranías unipersonales y de presión redemocratizadora en toda América Latina en los 50, que lleva directamente en la región de Centroamérica y el Caribe a las muertes del dictador nicaragüense Anastasio Somoza García y del general Leonides Trujillo de República Dominicana, a los derrocamientos en Venezuela de Andrés Pérez Jiménez y al fin de la dictadura de Fulgencio Batista en Cuba. 
En este contexto sociopolítico, hay que analizar las formas de supervivencia de las culturas étnicas salvadoreñas, y en especial la pipil después de la masacre de 1932 con base en preguntas claves como las siguientes:

- Enigma demográfico: ¿Es El Salvador un país mestizo?

- ¿Qué ocurre con el idioma náhuat?

- ¿Qué ocurre con las formas de organización familiar y con los modos de educación en general?

- Lo que eran los modos de la cultura material indígena y modernización (por ejemplo, la "españolización” de los nombres y apellidos pipiles, la muerte del idioma náhuat).

La segunda parte de este trabajo se ocupa de los discursos de la identidad en El Salvador. A partir de este análisis se abordará el proceso de industrialización en El Salvador en los años cincuenta, en el que la misma oligarquía cafetalera deviene en burguesía industrial, generándose una concentración del poder económico particular. Esta hegemonía financiera y política es el origen de la polarización de las fuerzas sociales en el país, debido a la injusta distribución de la riqueza nacional.

Por lo que toca a la redefinición de la identidad cultural salvadoreña después de 1932, basándose en los discursos sobre la cultura nacional existentes, debe destacarse la necesidad de hacer un balance de la obra del pensador salvadoreño Alberto Masferrer (1865-1932), el "Maestro de la juventud" salvadoreño, ya que es el único pensador que deja una obra sobre una moderna identidad cultural salvadoreña. Influido por ideas orientalistas (el budismo y la actitud contemplativa indú), así como por el laborismo inglés (fue ministro de cultura varios meses en 1931 bajo el gobierno del presidente laborista salvadoreño Arturo Araujo), su obra presenta para la realidad salvadoreña una propuesta social mezcla de reformismo contemplativo y resignación social.

Su doctrina del "Minimum vital" propone que cada ser humano se conforme con lo escencialmente necesario para vivir ("un cuarto limpio con una ventana donde entre el sol") y declara la guerra a "los vicios nacionales" como el alcoholismo, la prostitución, el bandidaje e incluso hasta el comunismo. Enemigo del progreso material (Dinero maldito) su propuesta se encamina hacia un orden contemplativo y pacifista en la línea de Mahatma Gandhi (Pan o revólver).

Tales ideas no consiguieron echar pie en la realidad salvadoreña. La oligarquía las rechazó y a la vez fueron blanco de la crítica de intelectuales de izquierda, como Pedro Geoffroy Rivas ("Mi Alberto Masferrer") y Roque Dalton ("Viejuemierda"). 
A partir de lo sucedido con Masferrer, debe señalarse la carencia de un discurso complejo acerca de la identidad cultural salvadoreña. No hay tampoco, por lo mismo, una articulación del problema de la multiplicidad de culturas y la heterogeinidad cultural.

En El Salvador el discurso del mestizaje no se articula teóricamente, como sí se articula a lo largo y ancho de América Latina a partir de los años treinta con obras como El laberinto de la soledad, de Octavio Paz; Siete ensayos de interpetación de la realidad peruana, de José Carlos Mariátegui; Radiografía de la Pampa, de Ezequiel Martínez Estrada; El Nicaragüense, de Pablo Antonio Cuadra; o de Guatemala, las líneas de su mano, de Luis Cardoza y Aragón. Mi hipótesis es que la carencia de un estudio que articule el mestizaje salvadoreño tiene que ver con la ausencia de una universidad modernizada, con la ausencia de una vida cultural estabilizada y con la ausencia de una tradición cultural que enfrente el problema sociocultural que surge a partir de 1932.

Esta carencia tambíen se debe a que la oligarquía y burguesía salvadoreñas, así como los principales actores sociales del país, como el Ejército títere, no necesitaron de discursos articulados en este terreno para afianzar su hegemonía, sino que les bastaron discursos generales para sostenerse, pues, luego del etnocidio de 1932, en El Salvador reina una especie de "paz de los cementerios" por más de cincuenta años bajo la hégida de la bota de las tiranías militares prooligárquicas.

Dentro del tratamiento de estas "carencias", también es necesario detenerse en el etnólogo alemán Leonhard Schultze Jena para especificar cuáles fueron las condiciones de su trabajo de campo en El Salvador (su ruta, sus informantes, su estadía de tres meses en Izalco) y los presupuestos científicos que le sirven para construir la gramática del idioma pipil (por ejemplo, al desconocer a Ferdinand de Saussure, se regía por un esquema clásico-ortodoxo basándose en sus conocimientos enciclopédicos de los idiomas clásicos como el arameo, el griego, el hebreo y el latín); explicar cómo recopila la serie de mitos, leyendas, tradiciones, usos y costumbres de los pipiles de Izalco, así como su entendimiento de las culturas indígenas centroamericanas, valiéndose de sus diferentes ensayos y trabajos científicos, escritos al respecto (por ejemplo: "Investigaciones etnológicas y lingüísticas en las tierras altas de Guatemala" o "Indianisches Sprachgut im Gebrauch bei den Europäern"; explicar la importancia de Indiana II. Mythen in der Muttersprache der Pipil von Izalco in El Salvador, como posible obra capaz de comprender las dimensiones de lo étnico dentro de lo que podría ser una cultura salvadoreña y centroamericana.

¿Por qué no pudieron tener eco ni recepción de ninguna especie en El Salvador los trabajos de Schultze Jena? ¿Por qué se le ignora después? ¿Qué pasó con sus informantes de 1930? Estas preguntas se relacionan con la ausencia del interés por su obra. Contribuye al desinterés y desconocimiento del libro de Schultze Jena el que este sea publicado en Europa, bajo condiciones especiales en un 


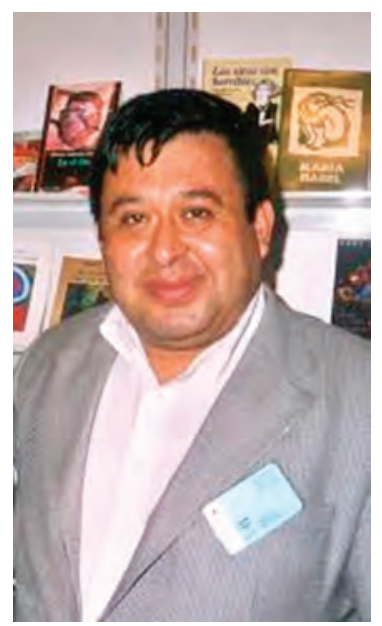

Dr. David Hernández

momento político muy álgido y en los comienzos de una “Alemania nazi”. Junto con el desconocimiento de Indiana Pipil también existe el desconocimiento en El Salvador de la continuación de la obra de Schultze Jena realizada posteriormente por científicos como Lyle Campbell (Diccionario Pipil) y en menor medida de William Fowler Jr. (El Salvador: Antiguas civilizaciones).

Por otro lado, volviendo a la década de los cincuenta, si se sale de la idea de los movimientos intelectuales generacionales y se trata de analizar la realidad latinoamericana en términos de procesos generales, la pregunta básica es: ¿Qué procesos generales tienen lugar en los años 50 en toda América Latina?

En dicha década se experimentan procesos generales de redemocratización unidos a procesos acelerados de modernización en América Latina en general y en El Salvador en particular. ¿Cuáles son los procesos de redemocratización a que ya aludimos? Son los que se llevan en la década de los cincuenta al final de gobiernos autoritarios o al final de gobiernos de tipo oligárquicopatriarcal en toda América Latina. El de Odría en el Perú; el de Pérez Jiménez, en Venezuela; el de Juan Domingo Perón, en la Argentina; el de Rojas Pinilla, en Colombia; el del gran populista Getulio Vargas, en el Brasil.

En Centroamérica esto se enmarca en el intento fallido de los democrátas nicaragüenses de un cambio social con la muerte de Anastasio Somoza García. Todos estos procesos de redemocratización tienen su colofón, alcanzan su cima, con el triunfo de las fuerzas armadas rebeldes de Fidel Castro Ruz contra la tiranía de Fulgencio Batista en Cuba en 1959. Estos procesos políticos están directamente emparentados con la nueva dinámica económica.

La industrialización en El Salvador "despega" con la "Revolución de 1948”, el movimiento reformista de la oligarquía y los militares. En este contexto acaece un fenómeno muy atípico en la sociedad salvadoreña, como es el hecho de que 
la misma oligarquía caficultora terrateniente, rural y semifeudal, aprovechando la coyuntura internacional de los excelentes precios del café en el mercado mundial, se transforma en burguesía industrial, urbana y capitalista.

De esta forma, en El Salvador no se experimentan las contradicciones antagónicas de clase, entre la oligarquía rural semifeudal y la burguesía urbana capitalista, que son propias en general para estos cambios estructurales en la sociedad. Eliminando las contradicciones entre una vieja oligarquía terrateniente cafetalera y una nueva burguesía industrial, el status quo salvadoreño mantiene en el poder hegemónico al mismo grupo de poderosas familias ("las catorce familias" o "los barones del café") que detentan el monopolio del poder económico, social y cultural en El Salvador.

En la tercera parte de esta investigación se trata la problemática de la supervivencia, de la nueva definición y de la reinvención de los elementos de las culturas indígenas, sus instituciones sociales y sus huellas lingüísticas.

En la década de los cincuenta surge en El Salvador un campo cultural relativamente autónomo, que conlleva el afianzamiento de una serie de subsistemas que se modernizan. En primer lugar surge un subsistema de enseñanza y de formación, que quiere decir Universidad Nacional, escuelas superiores, academia militar modernizada, mejoramiento de la escuela secundaria, enseñanza bilingüe (español e inglés), que repercutirá en las reformas educativas del país comenzadas en los años cincuenta y que tienen su coronación en la Reforma Educativa del período 1967-1971. En segundo lugar, en la década de los cincuenta surge un subsistema de artes, esto quiere decir que aparecen editoriales independientes, revistas, periódicos, nuevos grupos de artistas que se alejan de la bohemia, que ya no practican "l'art pour l'art" sino se constituyen en "inteligentzia": intelectuales militantes comprometidos en las luchas sociales de las fuerzas democráticas salvadoreñas.

Estos grupos, que tienen su cristalización en la "Generación comprometida" de 1956, van a darse: una estructura propia; van a inventarse y a otorgarse una geneología en base a preguntas como: ¿Quiénes son nuestros antecesores? ¿Quién es Salarrué? ¿De dónde venimos?; se harán un panorama de interlocutores internacionales. Ellos son los primeros que se plantean el problema de la identidad nacional. La "búsqueda del alma salvdoreña" (a través de estudios etnológicos en México o del rescate de la mitología pipil), en realidad no es ninguna búsqueda sino "la invención" o la "construcción", el "montaje" de las piezas básicas dadas por la tradición oral y el inconsciente colectivo, de la identidad nacional.

En este sentido, el papel que le dan al elemento indígena es de vital importancia, sobre todo el enfoque que toman a partir de los elementos que tienen, a partir de los cuales inventan, construyen, recolectan o estructuran el bajage cultural propio y colectivo.

El examen de las reinvenciones de los elementos culturales indígenas entre 1959-1979 exige pormenorización a partir del triunfo de la Revolución cubana en 1959. Lo ocurrido con las reinvenciones y la documentación de 
los elementos culturales indígenas en la literatura, la política y la sociedad en general, llevados a cabo por las capas intelectuales urbanas de El Salvador, y en especial las realizadas por el "Círculo Cultural Universitario" en 1956 dió origen a la "Generación comprometida". Varios de sus miembros, como Manlio Argueta y Roque Dalton, se cuentan entre los autores más representativos de la literatura salvadoreña del siglo XX. La "Generación comprometida" trató de reinventar la nacionalidad y de redefinir la identidad cultural salvadoreña con una literatura contestataria acompañada de una militancia política de izquierda ("apoyo combatiente" a la Revolución cubana y defensa contra la "agresión imperialista").

A la vez, introdujo elementos de heterogeneidad cultural, subvirtiendo de esta forma el canon de la ciudadanía salvadoreña, y al mismo tiempo, ampliándolo. Pues la ciudadanía salvadoreña en la década de los cincuenta se definía como españolaparlante, católica de religión y mestiza de "raza" (una lengua, una religión, una raza). Sin embargo, los escritores mencionados introducen en la literatura salvadoreña elementos indígenas, heterogeneidad que subvierte y da un vuelco a la definición de la nacionalidad. Al nivel de la escritura, finalmente, a través de la cristalización de elementos culturales míticos, apuntaron hacia una redefinición de los modos de existencia humana en medio de un acelerado proceso de modernización socioeconómica, política y cultural.

Como propuesta para una invención de lo nacional debe destacarse un corpus básico constituido por Tierra azul donde el venado cruza, un libro de poemas de Oswaldo Escobar Velado escrito en los años 50 y publicado en 1997; Cenizas de Izalco, novela escrita por Claribel Alegría y Darwin J. Flakoll en París entre 1960 y 1961 y publicada en Barcelona por Seix Barral en 1964; El Salvador, de Roque Dalton, estudio sociológico y antropológico publicado en La Habana en 1963.

Estas tres obras - un libro de poemas que rescata el elemento campesino-indígena de El Salvador y Centroamérica; una novela con un nombre emblemático, así como una monografía sociohistórica sobre El Salvador que intenta "buscar" unas raíces precolombinas y mitológicas del país - articulan la invención de la identidad cultural salvadoreña en la década de los cincuenta. En estas tres obras se encuentran las huellas y los signos de lo indígena. Fundan un discurso moderno sobre lo nacional y la nación en El Salvador en donde, de una manera u otra, vuelven a lo indígena.

En los sesenta los escritores de la "Generación comprometida" acrecientan ese corpus con una serie de textos. Entre ellos se destacan: El Valle de las Hamacas, novela de Manlio Argueta que busca una reinvención de El Salvador a partir de la época de la Conquista. El título de la novela es el nombre con el cual los conquistadores españoles conocían a la capital del país, San Salvador, debido a sus frecuentes terremotos. La novela fue publicada por la editorial "Sudamerica" de Argentina, en 1970. 
Además, Las historias prohibidas del Pulgarcito, un libro de poemas-collage de Roque Dalton, editado en México en 1974. En este poemario se hace un recorrido histórico de El Salvador, partiendo de la resistencia indígena pipil en la región de los izalcos en 1524 y terminando con la resistencia armada de la guerra de guerrilla en El Salvador de los primeros años de la década de los setenta. Lo notable del caso es que los mitos y elementos culturales recopilados por Schultze Jena son detectables en los textos de ficción de autores salvadoreños que no han podido leer esos materiales existentes en alemán.

Al enfocar el estado de guerra civil que experimentó el país en las dos décadas últimas, así como de las diferentes preocupaciones literarias y del pensamiento social por la articulación de lo indígena dentro del discurso de la invención de la identidad cultural salvadorena, las novelas de Manlio Argueta proporcionan material central. Entre ellas está Cuzcatlán, donde bate la mar del sur, novela editada en Costa Rica en 1987, que abarca una mirada histórica, desde la época precolombina hasta la guerra civil, sobre El Salvador. También se revisará la acción del grupo "La Masacuata", de finales de la década de los sesenta y principios de los setenta, cuyos miembros, todos poetas, se suman a la lucha política, lo cual impidió su desarrollo literario.

También el grupo "La Cebolla Púrpura" fundado en abril de 1971, cuyos miembros tienen los más disimiles destinos: Jaime Suárez (1950-80) es asesinado por los escuadrones de la muerte en San Salvador; Rigoberto Góngora (1950-82) cae como combatiente del Frente Farabundo Martí para la Liberación Nacional en medio de la guerra civil en Guazapa; y David Hernández (1955), luego de una estadía de siete años en la ex-Unión de Repúblicas Socialistas Soviéticas, termina instalándose en Europa.

Al analizar finalmente el estado sociopolítico del país después de la guerra, con la reconstrucción nacional, una de las cosas que se destaca, en las actuales circunstancias, es la revisión del canon cultural salvadoreno. Dentro de esta redefinición, el presente artículo aboga para que se introduzca, a un nuevo nivel teórico y político-cultural en la actual discusión cultural, por fin un elemento clave, decisivo: el elemento cultural indígena de origen pipil, herencia cultural que ha sido recogida y editada en alemán por Schultze Jena.

Se imponen, como imperativos, la divulgación de la obra Indiana Pipil, así como la discusión en torno a los elementos culturales de los izalcos recogidos en dicha obra. A este propósito queremos retener la propuesta de Reinhold Görling:

Debemos deconstruir las figuras de pensamiento del siglo XIX para conectarnos con lo que pueda haber todavía en ellas de validez. Y quizas no se trata tanto de figuras de pensamiento, de categorías, sino más bien de características propias, de principios éticos que estaban unidos con esas figuras 
de pensamiento pero que en parte también fueron recubiertos por ellas. Me refiero en el caso de los cosmopolitas a la curiosidad hacia lo extranjero, entre los nacionalistas se trata del reconocimiento de particularidades, entre los internacionalistas del principio de la solidaridad. Y todavía más importante es que se trata de elementos surgidos históricamente en contramovimientos y por lo tanto no pueden seguier siendo pensados como exclusividades. Curiosidad por lo extranjero: debe dejarse orientar por un reconocimiento del otro, sin transformar la diferencia en algo esencialista, en algo que solo corrobora la propia especificidad. La solidaridad, la percepción no solo de lo propio sino tambien de lo diferente como igual, presupone en nosotros al mismo tiempo curiosidad y un saber acerca de lo extranjero.[3]

El presente trabajo cobró vida a partir de la confrontación de mi experiencia de la cultura salvadoreña dentro y fuera de El Salvador con la lectura del libro de Schultze Jena sobre los pipiles de Izalco, escrito en 1931, antes de la hecatombe que puso en cuestión la existencia misma de la comunidad indígena. A partir de esa confrontación busqué desarrollar hipótesis científicas metodológicamente operacionables para bosquejar respuestas a una serie de preguntas que han servido de punto de arranque: acerca del destino de las culturas indígenas en El Salvador; del valor y las limitaciones de las explicaciones formuladas sobre las bases para la necesaria revisión del desarrollo histórico-social del país a partir de dicha fecha hasta nuestros días; acerca de las formas cómo los principales escritores de El Salvador consiguen o no dar cabida a la cultura indígena en sus textos. También fue necesario intentar explicar impresionantes vacíos en el discurso cultural salvadoreño, lo que nos llevó a revaluar textos de muy distintos géneros leídos aquí desde una perspectiva de análisis del discurso.

Para llevar adelante la investigación, me han sido de mucha utilidad trabajos investigativos parciales y proyectos de edición que había adelantado en el transcurso de los últimos diez años. Entre ellos está la antología de poesía; Pobrecito pulgarcito poeta país. 200 años de poesía en El Salvador (terminada en 1994, parcialmente traducida en francés por la Univesidad de Perpignon, Francia), así como diferentes estudios sobre la novelística de Manlio Argueta.

Un primer bosquejo general de las hipótesis aquí desarrollada y los resultados investigativos alcanzados aparece en el ensayo "El Salvador, exilio y literatura", publicado en diferentes medios de comunicación y libros en El Salvador, Estados Unidos y Alemania. Debo agregar también que me ha sido muy útil mi trabajo en el periodismo cultural, en particular para diarios de los Estados Unidos, ante todo La Opinión, de Los Ángeles, y, más recientemente, para publicaciones de El Salvador.

La bibliografía usada en este trabajo se encuentra en gran parte en la biblioteca del Instituo Iberoamericano de Berlin, la más importante de Europa en lo que a fondos latinoamericanos se refiere, y cuyos fondos de hemeroteca y biblioteca sobre El Salvador son más ricos que los disponibles en este país. En este sentido, este trabajo es también deudor de la riqueza bibliográfica existente en esta importante institución, que viene prestando invaluables servicios a la 
investigación sobre América Latina. Expreso, así mismo, mis agradecimientos a diferentes instituciones que de una $\mathrm{u}$ otra forma me han apoyado para la realización de esta tesis, en especial a la Universidad de Hannover.

También a los directores científicos de este trabajo: Prof. Dr. Reinhold Görling, de la Universidad de Hannover; y de manera muy especial al Prof. Dr. Carlos Rincón, del Lateinamerika-Institut der Freien Universität Berlin (Instituto Latinoamérica de la Universidad Libre de Berlín). Así mismo, debo agradecer a mis compañeros de estudio de la Universidad de Hannover del coloquio interdisciplinario para doctorantes, por la discusión de algunos aspectos de nuestras investigaciones científicas y sus valiosas opiniones.

\section{Notas}

[1] Ver: Comisión de la Verdad, 1993. De la locura a la esperanza. La guerra de doce años en El Salvador. Informe de la Comisión de la Verdad. San Salvador, Editorial Arcoiris.

[2] Schlesinger, Jorge. 1946. Revolución comunista. ¿Guatemala en peligro?, Ciudad de Guatemala, Unión Tipográfica Castañeda, Ávila y Cía.

[3] Görling, R., Heterotopía, Pág. 9. 


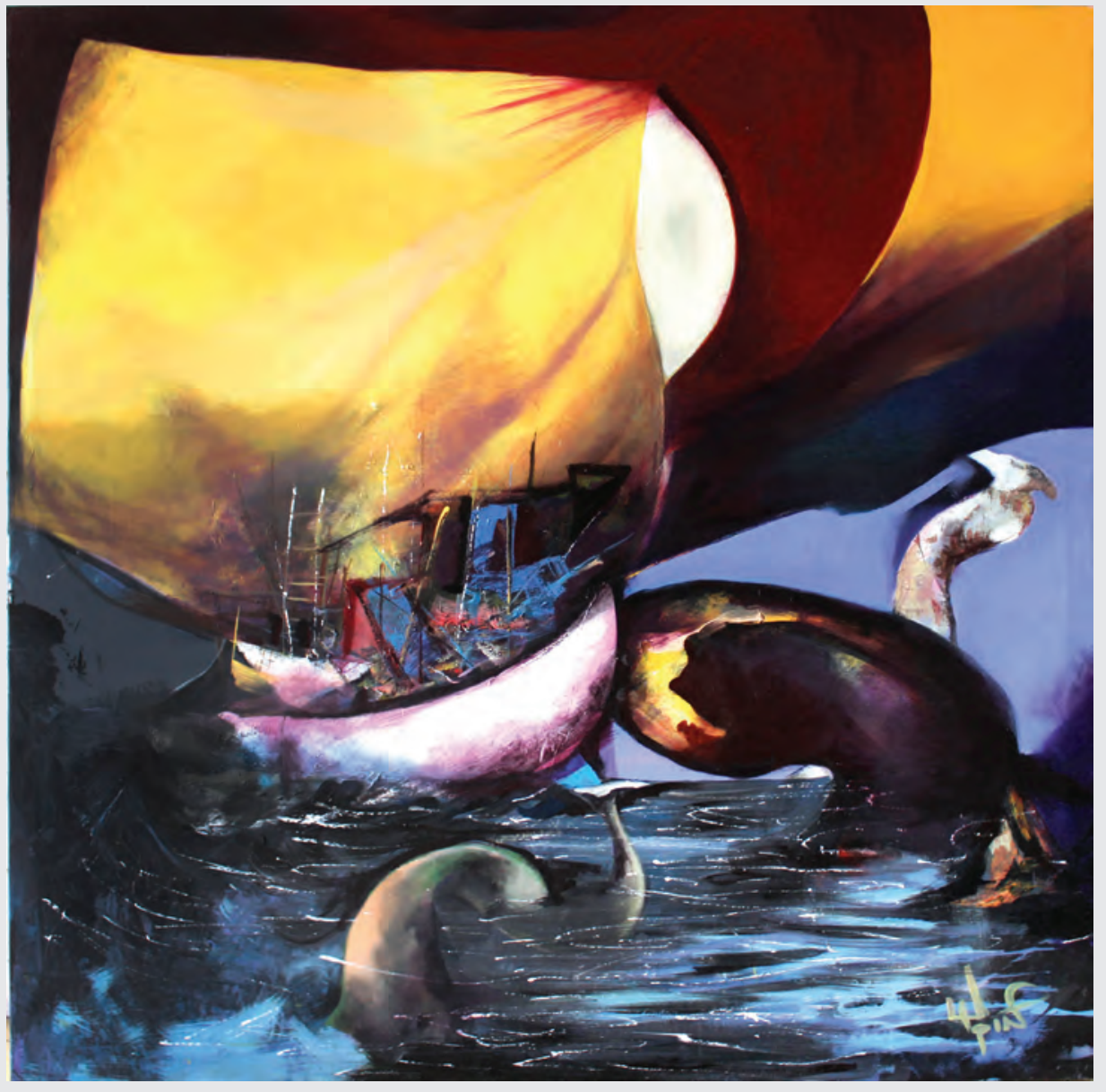

"Viajero de mil mares"

Medidas: 146 x $147 \mathrm{cms}$

Técnica: mixta

Año: 2010 